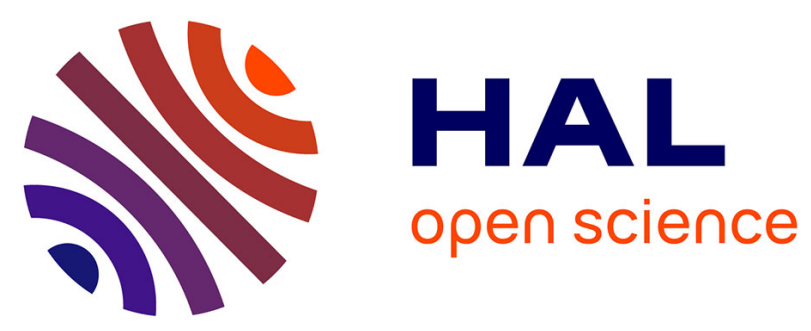

\title{
Theoretical and experimental study of an harmonically forced vibro-impact nonlinear energy sink
}

\author{
Etienne Gourc, Guilhem Michon, Sébastien Seguy, Alain Berlioz
}

\section{To cite this version:}

Etienne Gourc, Guilhem Michon, Sébastien Seguy, Alain Berlioz. Theoretical and experimental study of an harmonically forced vibro-impact nonlinear energy sink. ASME International Design Engineering Technical Conferences \& Computers and Information in Engineering Conference, DETC2013-13172, 4-7 août 2013, Portland, OR, États-Unis, Aug 2013, Portland, OR, United States. hal-02183338

\section{HAL Id: hal-02183338 \\ https://hal.science/hal-02183338}

Submitted on 5 Jul 2021

HAL is a multi-disciplinary open access archive for the deposit and dissemination of scientific research documents, whether they are published or not. The documents may come from teaching and research institutions in France or abroad, or from public or private research centers.
L'archive ouverte pluridisciplinaire HAL, est destinée au dépôt et à la diffusion de documents scientifiques de niveau recherche, publiés ou non, émanant des établissements d'enseignement et de recherche français ou étrangers, des laboratoires publics ou privés. 


\section{DETC2013-13172}

\section{THEORETICAL AND EXPERIMENTAL STUDY OF AN HARMONICALLY FORCED VIBRO-IMPACT NONLINEAR ENERGY SINK}

\author{
Etienne Gourc* \\ Université de Toulouse \\ Institut Clément Ader, INSA \\ F-31077, Toulouse, France \\ Sébastien Seguy \\ Université de Toulouse \\ Institut Clément Ader, INSA \\ F-31077, Toulouse, France
}

\author{
Guilhem Michon \\ Université de Toulouse \\ Institut Clément Ader, ISAE \\ F-31055, Toulouse, France
}

\author{
Alain Berlioz \\ Université de Toulouse \\ Institut Clément Ader, UPS \\ F-31062, Toulouse, France
}

\begin{abstract}
Recently, it has been demonstrated that a Vibro-Impact type Nonlinear Energy Sink (VI-NES) can be used efficiently to mitigate vibration of a Linear Oscillator (LO) under transient loading. In this paper, the dynamic response of an harmonically forced LO, strongly coupled to a VI-NES is investigated theoretically and experimentally. Due to the small mass ratio between the LO and the flying mass of the NES, the obtained equation of motion are analyzed using the method of multiple scales in the case of $1: 1$ resonance. It is shown that in addition to periodic response, system with VI-NES can exhibit Strongly Modulated Response (SMR). Experimentally, the whole system is embedded on an electrodynamic shaker. The VI-NES is realized with a ball which is free to move in a cavity with a predesigned gap. The mass of the ball is less than $1 \%$ of the mass of the LO. The experiment confirms the existence of periodic and SMR response regimes. A good agreement between theoretical and experimental results is observed.
\end{abstract}

*e-mail: gourc@insa-toulouse.fr

\section{INTRODUCTION}

Targeted Energy Transfer (TET), also called energy pumping has been widely studied during the last decade. It has been demonstrated that the addition of small mass (compared to the mass of the main system) oscillator with essential stiffness nonlinearity can lead to efficient vibration absorption. Such a device is referred as Nonlinear Energy Sink (NES).

Energy pumping under transient loading has been extensively studied. In [1,2], it has been shown that the main phenomena allowing TET is based on the $1: 1$ resonance capture. Experimental verification are presented in [3-5].

TET under external forcing has also been studied. Introduction of a suitable asymptotic procedure based on the invariant manifold approach [6] has shown that in addition to periodic regimes, system with NES can exhibit beating response, referred as Strongly Modulated Response (SMR) [7]. This type of response has been verified experimentally in [8]. The use of NES to passively control instability is also a growing interest. In [9], a NES is used to control limit cycle oscillation of a Van der Pol system. [10-13] studied the effectiveness of a NES to suppress aeroelastic instability. In [14], it is shown that a NES can be used 
to control chatter instability in turning.

All the aforementioned studies deal with NES with cubic nonlinearity. Recent studies have enlighten that non-smooth system can be used as NES. One of the main advantage of this type of NES over classic NES is that they are often easier to build than classic NES. Gendelman investigated energy transfer in system with non-polynomial nonlinearity [15]. NES with piecewise piecewise linear stiffness have been studied in [16] under transient and periodic forcing. The case of a vertical NES, considering its own weight has been reported in [17].

Vibro-impact type NES (VI-NES) have been studied in [18-20]. However, these study were concentrated around numerical simulations. In a recent study, the invariant manifold approach has been extended to VI-NES under transient loading [21].

In this paper an harmonically forced Linear Oscillator (LO) with an embedded VI-ENS is considered theoretically, using the invariant manifold approach and experimentally. The behavior of the system is studied in the regime of $1: 1$ resonance. The structure of the paper is as follow. Section 2, is devoted to the analytic treatment of the governing equation of motion. In section 3, analytic results are presented and are compared to numerical simulation. In the next section, the experimental setup is presented. Measurements are compared to analytical and numerical results. Finally, concluding remarks are addressed.

\section{THEORETICAL DEVELOPMENTS}

The system considered is composed of a Linear Oscillator (LO) with an embedded Vibro Impact Nonlinear Energy Sink (VI-NES). The LO is subjected to an imposed base displacement. The system is presented in Fig. 1. The reduced governing equation of motion, between impact, are expressed as:

$$
\begin{gathered}
\ddot{x}_{1}+\varepsilon \lambda \dot{x}_{1}+x_{1}=\varepsilon A \sin \Omega t+\varepsilon^{2} \lambda A \cos \Omega t \\
\ddot{x}_{2}=0, \quad \forall\left|x_{1}-x_{2}\right|<\Delta
\end{gathered}
$$

where

$$
\begin{gathered}
\varepsilon=\frac{m_{2}}{m_{1}}, \quad \omega_{1}=\frac{k_{1}}{m_{1}}, \quad \lambda=\frac{c_{1}}{m_{2} \omega_{1}}, \\
A=\frac{G}{\varepsilon}, \quad \Omega=\frac{\omega}{\omega_{1}}, \quad t=\omega_{1} \tilde{t}
\end{gathered}
$$

$x_{1}$ and $x_{2}$ are the displacement of the LO and the NES respectively and the dots denotes differentiation with respect to the non-dimensional time $t$.

When $\left|x_{1}-x_{2}\right|=\Delta$, a collision occurs. The state of the system after impact is obtained using the simplified shock theory and the condition of total momentum conservation:

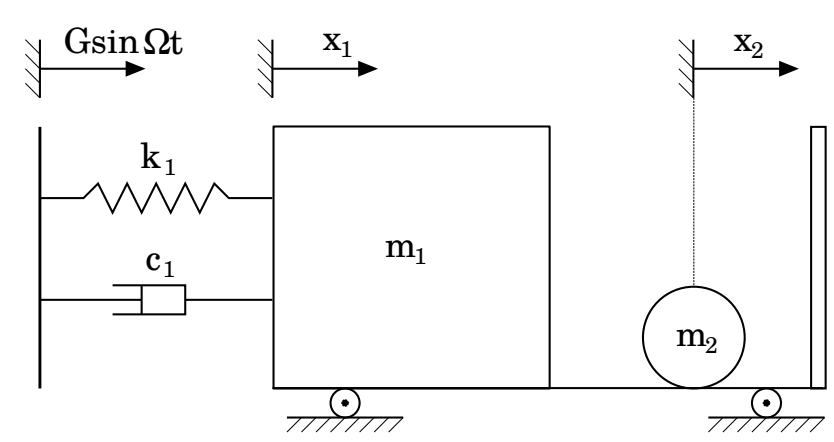

FIGURE 1. Schema of the system

$$
\begin{gathered}
x_{1}^{+}=x_{1}^{-}, \quad x_{2}^{+}=x_{2}^{-} \\
\dot{x}_{1}^{+}+\varepsilon \dot{x}_{2}^{+}=\dot{x}_{1}^{-}+\varepsilon \dot{x}_{2}^{-}, \quad \dot{x}_{1}^{+}-\dot{x}_{2}^{+}=-r\left(\dot{x}_{1}^{-}-\dot{x}_{2}^{-}\right), \\
\text {for }\left|x_{1}-x_{2}\right|=\Delta
\end{gathered}
$$

where $r$ is the restitution coefficient of impact and the superscripts + and - denotes time immediately after and before impact. New variables representing the displacement of the center of mass and the internal displacement of the VI-NES are introduced as follow:

$$
v=x_{1}+\varepsilon x_{2}, \quad w=x_{1}-x_{2}
$$

Substituting Eq. (3) into Eqs. (1) and (2), the equation between impact in barycentric coordinate are given as:

$$
\begin{gathered}
\ddot{v}+\varepsilon \lambda \frac{\dot{v}+\varepsilon \dot{w}}{1+\varepsilon}+\frac{v+\varepsilon w}{1+\varepsilon}=\varepsilon A \sin \Omega t+\varepsilon^{2} \lambda A \cos \Omega t \\
\ddot{w}+\varepsilon \lambda \frac{\dot{v}+\varepsilon \dot{w}}{1+\varepsilon}+\frac{v+\varepsilon w}{1+\varepsilon}=\varepsilon A \sin \Omega t+\varepsilon^{2} \lambda A \cos \Omega t \\
\forall|w|<\Delta
\end{gathered}
$$

And the impact condition (2) is rewritten as:

$$
\begin{gathered}
v^{+}=v^{-}, \quad w^{+}=w^{-}, \\
\dot{v}^{+}=\dot{v}^{-}, \quad \dot{w}^{+}=-r \dot{w}^{-}, \quad \text { for }|w|=\Delta
\end{gathered}
$$

In the context of energy pumping, the mass ratio $\varepsilon$ is supposed to be small $(\approx 1 \%)$. In this case, Eq. $(4)$ may be analyzed by multiple scales approach with respect to this small parameter. Multiple scales are introduced in the form: 


$$
\begin{gathered}
v(t ; \varepsilon)=v_{0}\left(T_{0}, T_{1}\right)+\varepsilon v_{1}\left(T_{0}, T_{1}\right)+\ldots \\
w(t ; \varepsilon)=w_{0}\left(T_{0}, T_{1}\right)+\varepsilon w_{1}\left(T_{0}, T_{1}\right)+\ldots \\
T_{k}=\varepsilon^{k} t, \quad k=0,1, \ldots
\end{gathered}
$$

We are interested in the behavior of the system in the vicinity of the $1: 1$ resonance where the LO oscillates with a frequency close to external forcing while the VI-NES operates with two symmetric impact per cycle. A detuning parameter $(\sigma)$ representing the nearness of the excitation frequency $\Omega$ to the reduced natural frequency of the LO is introduced:

$$
\Omega=1+\varepsilon \sigma
$$

Substituting Eqs. (6) and (7) into Eqs. (4), (5) and equating coefficients of like power of $\varepsilon$ gives:

Order $\varepsilon^{0}$ :

$$
\begin{gathered}
D_{0}^{2} v_{0}+v_{0}=0 \\
D_{0}^{2} w_{0}+v_{0}=0, \quad \forall\left|w_{0}\right|<\Delta \\
D_{0} v_{0}^{+}=D_{0} v_{0}^{-}, \quad v_{0}^{+}=v_{0}^{-}, \quad w_{0}^{+}=-r D_{0} w_{0}^{-}, \quad \text { for }\left|w_{0}\right|=\Delta
\end{gathered}
$$

Order $\varepsilon^{1}$ :

$$
D_{0}^{2} v_{1}+v_{1}=-2 D_{0} D_{1} v_{0}-\lambda D_{0} v_{0}-w_{0}+v_{0}+A \sin \left(T_{0}+\sigma T_{1}\right)
$$

Analytic treatment of $O\left(\varepsilon^{0}\right)$ equation is based on [21]. The first equation of system (8), taking into account Eq. (9) simply represents an undamped harmonic oscillator and its solution can be expressed as follow:

$$
v_{0}=C\left(T_{1}\right) \sin \left(T_{0}+\theta\left(T_{1}\right)\right)
$$

The second equation of system (8) with (9) represents an harmonically forced impact oscillator with symmetric barrier. Under the assumption of $1: 1$ resonance and motion with two symmetric impact per cycle, its solution can be searched in the following form [21]:

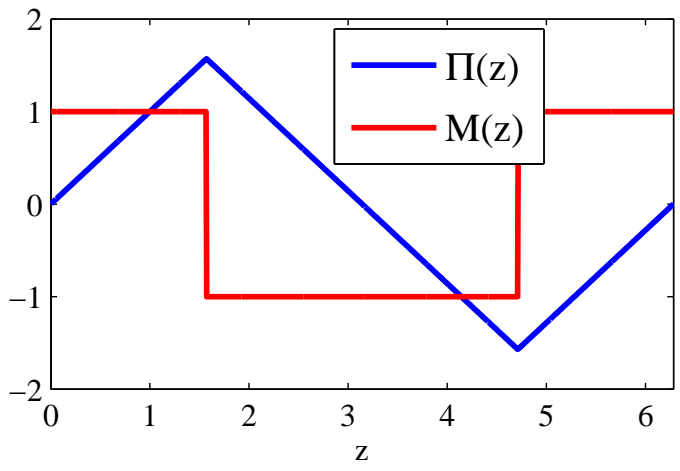

FIGURE 2. Representation of the non-smooth functions $\Pi(z)$ and $M(z)$

$$
w_{0}=C\left(T_{1}\right) \sin \left(T_{0}+\theta\left(T_{1}\right)\right)+\frac{2}{\pi} B\left(T_{1}\right) \Pi\left(T_{0}+\eta\left(T_{1}\right)\right)
$$

Where $\Pi(z)$ is a non-smooth zig-zag function. This folded function and its derivative is depicted in Fig. 2 and are expressed as follow:

$$
\Pi(z)=\arcsin (\sin z), \quad M(z)=\frac{d \Pi}{d z}=\operatorname{sgn}(\cos z)
$$

According to Eq. (12) and (13), impact occurs at $T_{0}=\pi / 2-$ $\eta+j \pi$ with $j=0,1,2, \ldots$ The impact condition $\left|w_{0}\right|=\Delta$ is rewritten with Eq. (12) as:

$$
C \cos (\eta-\theta)=\Delta-B
$$

Rewriting now the inelastic impact condition (9) yields:

$$
C(1+r) \sin (\eta-\theta)=\frac{2}{\pi} B(1-r)
$$

Combining Eqs. (14) and (15), a relation between the slow variables $B$ and $C$ is obtained as follow:

$$
C^{2}=\left(1+\frac{4(1-r)^{2}}{\pi^{2}(1+r)^{2}}\right) B^{2}-2 \Delta B+\Delta^{2}
$$




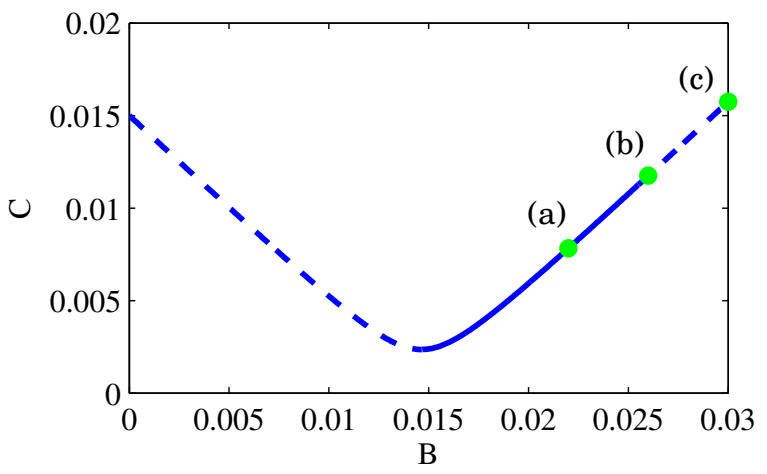

(a)

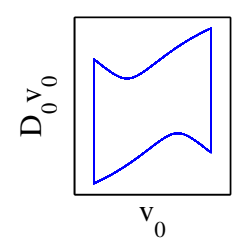

(b)

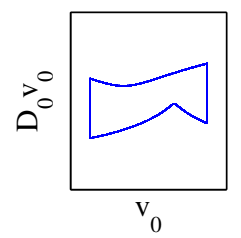

(c)

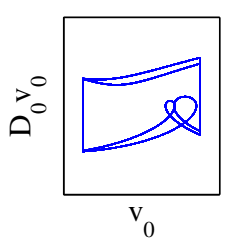

FIGURE 3. SIM of the problem for $r=0.6, \Delta=0.015$. Straight and dotted lines denotes stable and unstable branch of the SIM respectively. Numerical phase space : (a) stable symmetric $1: 1$ motion, (b) asymmetric $1: 1$ motion, (c) asymmetric $2: 2$ motion.

As it has been reported in [21], Eq. (16) defines the expression of the Slow Invariant Manifold (SIM) of the problem in the case of $1: 1$ resonance. An example of SIM is depicted in Fig. 3 for $\Delta=0.015$ and $r=0.65$. Note that the value of $r$ is a realistic value for the restitution coefficient in the case of steel-steel contact. As seen on Fig. 3, the SIM is divided in two branches. The stability of the SIM is evaluated by direct numerical integration of Eq. (9). The left part of the SIM is unstable, while a part of the right side is stable. As depicted on the phase space in Fig. 3, the $1: 1$ motion with two symmetric impact per cycle is stable until $B \approx 0.0255$. For higher values of $B$, the motion becomes asymmetric, then $1: 1$ symetric motion is refered as unstable. Further increase of $B$ can degenerate into motion with more than two impact per cycle. The extrema of the SIM is computed by equating the derivative of the right hand side of Eq. (16) to zero:

$$
\begin{gathered}
B_{1}=\frac{\Delta \pi^{2}(1+r)}{\pi^{2}(1+r)+4(1-r)} \\
C_{1}^{2}=\frac{4 \Delta^{2}(1-r)^{2}}{\pi^{2}(1+r)^{2}+4(1-r)^{2}}
\end{gathered}
$$

The value $C_{1}$ corresponds to the minimum amplitude of the LO which allow $1: 1$ TET.
In order to study the evolution of the system on the SIM, Eq. (10) at the next order of approximation is analyzed. To identify terms that produce secular terms, the function $\Pi(z)$ in Eq. (13) is expanded in Fourier series:

$$
\Pi(z)=\sum_{k=1}^{\infty} \frac{-4(-1)^{k}}{\pi(2 k-1)^{2}} \sin ((2 k-1) t)
$$

Substituting Eq. (11), (12) and (18) into Eq. (10) and eliminating terms that produce secular terms gives:

$$
\begin{array}{r}
-2 D_{1} C \cos \theta+2 C \sin \theta D_{1} \theta-\lambda C \cos \theta \\
-\frac{8}{\pi^{2}} B \sin \eta+A \sin \left(\sigma T_{1}\right)=0 \\
2 D_{1} C \sin \theta+2 C \cos \theta D_{1} \theta+\lambda C \sin \theta \\
-\frac{8}{\pi^{2}} B \cos \eta+A \cos \left(\sigma T_{1}\right)=0
\end{array}
$$

Rearranging in a more convenient form yields:

$$
\begin{gathered}
2 C D_{1} \theta=\frac{8}{\pi^{2}} B \cos (\eta-\theta)-A \cos \left(\sigma T_{1}-\theta\right) \\
2 D_{1} C=-\lambda C-\frac{8}{\pi^{2}} B \sin (\eta-\theta)+A \sin \left(\sigma T_{1}-\theta\right)
\end{gathered}
$$

Substituting Eqs. (14), (15) into Eq. (20) and introducing $\gamma=\sigma T_{1}-\theta$, the following equations are obtained:

$$
\begin{aligned}
2 C D_{1} \gamma & =-\frac{8 B(\Delta-B)}{\pi^{2} C}+A \cos \gamma+2 C \sigma \\
2 D_{1} C & =-\frac{16 B^{2}(1-r)}{\pi^{3} C(1+R)}+A \sin \gamma-\lambda C
\end{aligned}
$$

The fixed points are computed by equating the derivative of the right hand side of Eq. (21) to zero:

$$
\begin{aligned}
-\frac{8 B}{C \pi^{2}}(\Delta-B)+A \cos \gamma+2 C \sigma & =0 \\
-\frac{16 B^{2}(1-r)}{\pi^{3} C(1+R)}+A \sin \gamma-\lambda C & =0
\end{aligned}
$$

Using trigonometric identities and solving for $C^{2}$, new relations between $C$ and $B$ are obtained: 


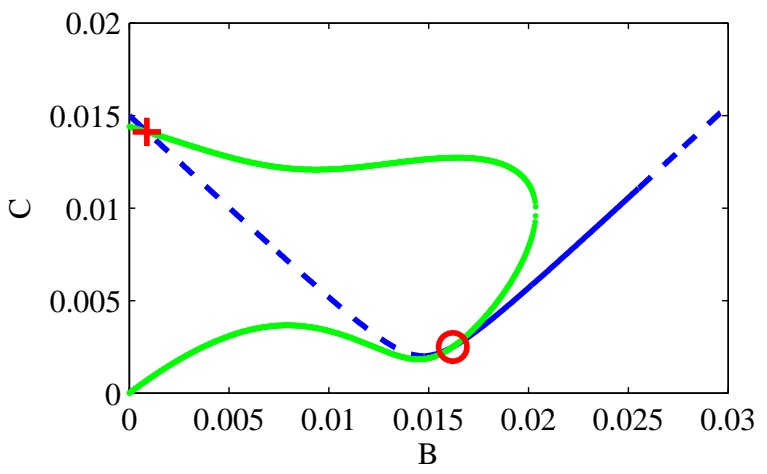

FIGURE 4. Case of stable periodic response. Blue and green lines corresponds to the SIM (16) and the curves (23). Red circle (o) and red cross (+) corresponds to stable and unstable fixed points respectively. Parameters are given in Eq. (24).

$$
C^{2}=b_{1} B, \quad C^{2}=b_{2} B
$$

Finally, the fixed points of the slow-flow can be obtained graphically as the intersection of the SIM (16), and the curve $b_{1}$ and/or $b_{2}$. Fixed points can also been expressed by equating the right hand sides of Eq. (16) and Eq. (23). A fourth order polynomial in $B$ is then obtained. The stability of the fixed points has not been analysed herein and will be studied in future developments.

\section{NUMERICAL SIMULATIONS}

In this section, various response regimes for different values of parameters are presented. Numerical simulations highlights the relation between the topology of the SIM, and the global behavior of the system.

In Fig. 4, the SIM (16), denoted by blue line, and the curves (23), denoted by green lines are presented for the following set of parameters:

$$
\begin{aligned}
\varepsilon=0.84 \%, & \lambda=0.95, \quad A=0.015 \\
\Delta=0.015, & r=0.65, \quad \sigma=-0.2
\end{aligned}
$$

At their intersection, red circle (o) and cross (+) represents stable and unstable fixed points respectively. In the present case, the green curves intersect the SIM two times. The fixed point located on the left side of the SIM is unstable, while the one located on the right branch of the SIM is stable.

If the initial condition are in the domain of attraction of the SIM, the flow will be automatically attracted to the stable fixed

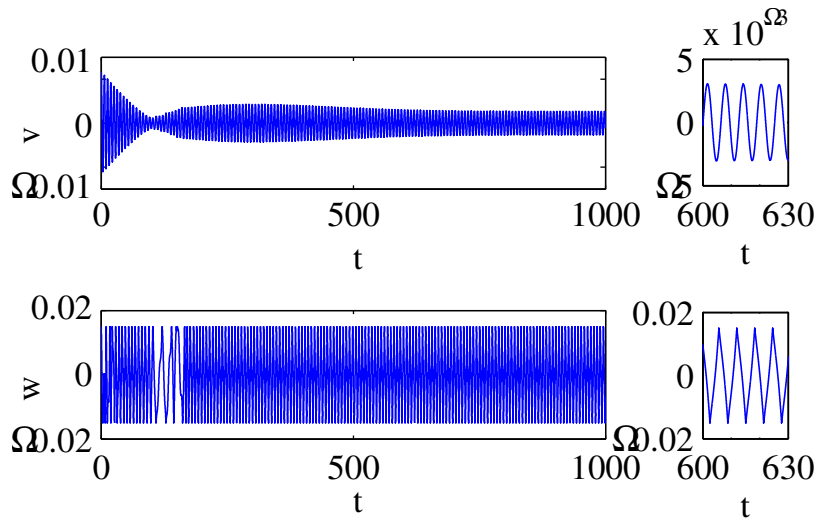

FIGURE 5. Numerical integration of Eqs. (4) and (5) for the set of parameters (24) and zoom on the response

points. The system will exhibit periodic motion with two symmetric impact per cycle. This prediction is confirmed by numerical integration of system (4), (5) depicted in Fig. 5. The displacement of the center of mass $(v)$ for parameters $(24)$ is presented. As expected from Fig. 4, after a short transient, the flow is rapidly attracted to the fixed points and stable periodic response is observed.

Another set of parameters where stable fixed points does not exist is now considered:

$$
\begin{gathered}
\varepsilon=0.84 \%, \quad \lambda=0.95, \quad A=0.012, \\
\Delta=0.015, \quad r=0.65, \quad \sigma=0
\end{gathered}
$$

The diagram of the slow-flow on the SIM is presented in Fig. 6 for the set of parameters (25). In this case two unstable fixed points are located on the lower branch of the SIM. The only possible response for the system is stable Strongly Modulated Response (SMR). This regime is observed on the numerical integration at Fig. 7. Contrary to NES with cubic stiffness $[7,14]$, where, during SMR, the flow jumps between two stable branches, in this case, it is observed that SMR act through successive synchronization between the LO and the VI-NES. When the VI-NES is not synchronized, the amplitude of the LO grows, under certain circumstance, the VI-NES enter in a $1: 1$ resonance capture with the LO. Then the amplitude of the LO decays until $C=C_{1}$ (17). Finally, the NES escapes resonance capture, and the amplitude of the LO starts growing again.

\section{EXPERIMENTAL TRIALS}

The experimental setup is depicted in Fig. 8(a). It consists of a LO, with an embedded VI-NES. The whole system is embedded on $10 \mathrm{kN}$ electrodynamic shaker. The displacement of the 


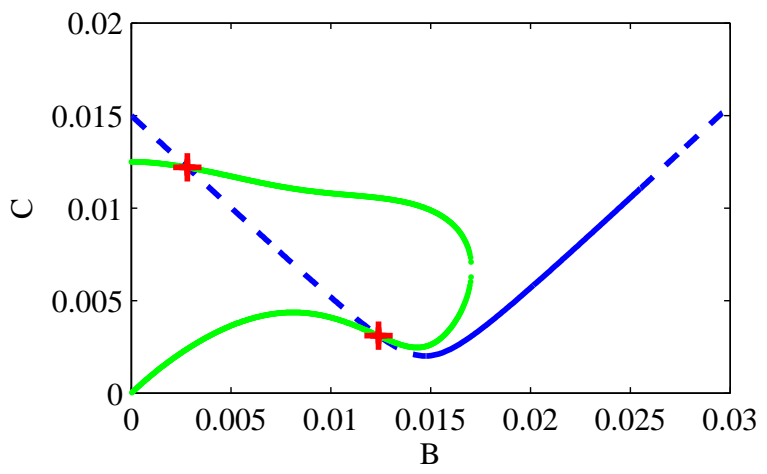

FIGURE 6. Case of SMR response. Blue and green lines corresponds to the SIM (16) and the curves (23). Red cross (+) corresponds to unstable fixed points. Parameters are given in Eq. (25).
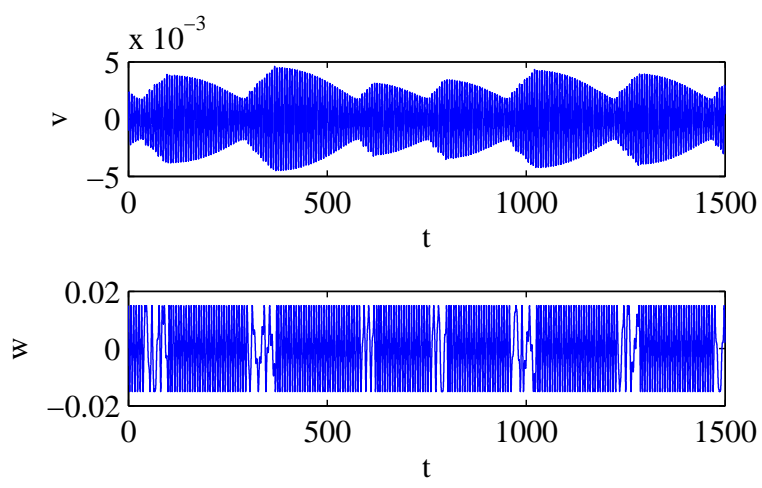

FIGURE 7. Numerical integration of Eqs. (4) and (5) for the set of parameters (25).

LO as well as the imposed displacement of the shaker are measured using contact-less laser displacement sensors. A detailed view of the VI-NES is presented in Fig. 8(b). It simply consists of a closed cavity of length $d+2 \Delta$, where $d$ is the diameter of the ball. Each cover is made of hardened steel. The design of the VI-NES is voluntary simple to check whether this kind of system can be used as vibration mitigation device. Note that the aim of this experiment is not to built an efficient NES, but to investigate the different response regimes for future exploration.

The parameters of the system have been identified by performing modal analysis and are summarized in Table 1.

Experimental trials have been carried out for three different forcing amplitudes. For each trials, displacement of the LO has been recorded for increasing and decreasing frequency around the natural frequency of the LO. The first results for $G=0.16 \mathrm{~mm}$ $(A=0.019)$ is depicted in Fig. 9. Blue lines are the analytical fixed points. Red dashed vertical lines represent the zone of SMR found using numerical integration of Eqs. (3), (4). Green

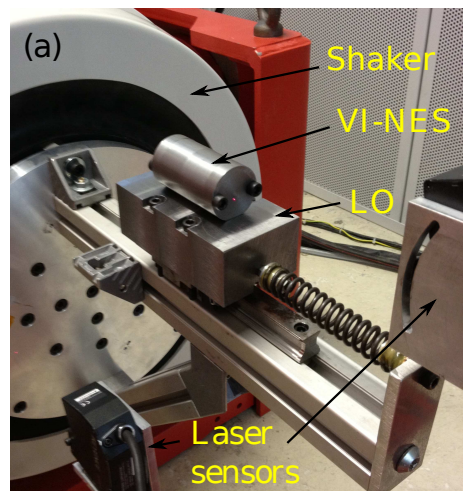

(b)

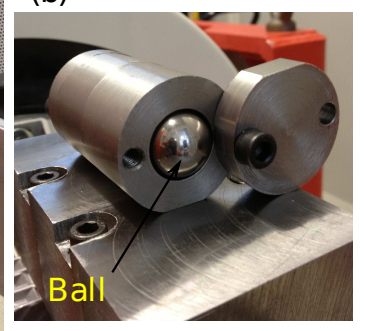

FIGURE 8. Picture of the experimental setup. (a) Global view of the system, (b) detailled view of the NES.

TABLE 1. Parameters of the experiment

\begin{tabular}{cc|cc}
\hline \multicolumn{4}{c}{ Physical Parameters } \\
\hline$m_{1}$ & $3.807 \mathrm{~kg}$ & $c_{1}$ & $2.53 \mathrm{Ns} / \mathrm{m}$ \\
$k_{1}$ & $11.68 * 10^{3} \mathrm{~N} / \mathrm{m}$ & $m_{2}$ & $32 \mathrm{~g}$ \\
$\Delta$ & $15 \mathrm{~mm}$ & $r$ & 0.6 \\
\hline \multicolumn{4}{c}{ Reduced Parameters } \\
\hline$\varepsilon$ & $0.84 \%$ & $\lambda$ & 1.43 \\
\hline
\end{tabular}

lines represents measured periodic response and straight vertical lines represents the experimentally determined zone of SMR. First periodic response were observed for $\sigma=-3.2$. This branch of periodic solution has been followed until $\sigma=1.1$. For further increase of the forcing frequency, periodic solution loose its stability, and SMR response takes place as illustrated in Fig. 3.

Experimentally, it has been found that stable SMR takes place between $\sigma=1.25$ and $\sigma=2.22$ versus $\sigma=1.6$ and $\sigma=3$ numerically. Similar results are obtained for a reduced forcing amplitude $(G=0.14 \mathrm{~mm}, A=0.017)$ and are depicted in Fig. 11. In this case, periodic motion is still observed between $\sigma=-2.08$ and $\sigma=0.42$. SMR response is also observed between $\sigma=0.55$ and $\sigma=1.8$ experimentally versus $\sigma=0.9$ and $\sigma=2.6$ numerically.

Decreasing the forcing amplitude to $G=0.125 \mathrm{~mm}$ ( $A=$ 0.015 ), the behavior of the system changes drastically and is presented in Fig. 12. For this case, a stable fixed does not exist, and SMR regimes are observed for a larger zone of forcing frequency. Experimentally, SMR has been observed from $\sigma=-1.94$ to $\sigma=1.39$, versus $\sigma=-2.2$ and $\sigma=2.4$ numerically.

This last trial is of particular interest since high amplitude fixed points are not observed anymore, and passive con- 


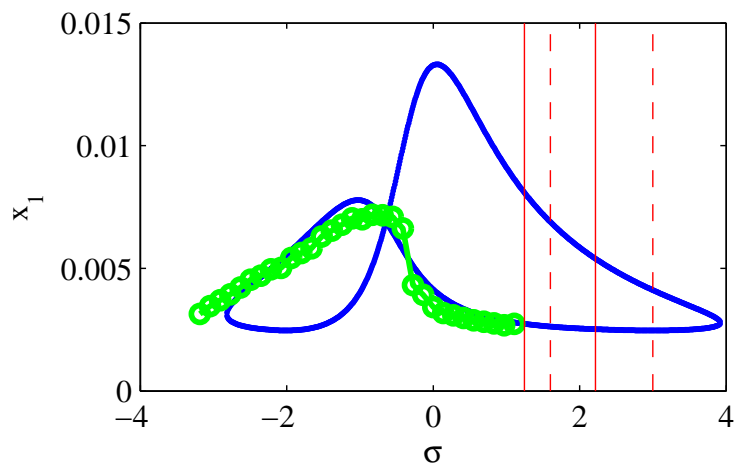

FIGURE 9. Experimental and analytical frequency response curves of the LO for $G=0.16 \mathrm{~mm}(A=0.019)$. Blue lines corresponds to the analytical prediction, green lines represents the experimental measurements. Straight and dashed red lines denotes the experimentally and numerically founded SMR zone.

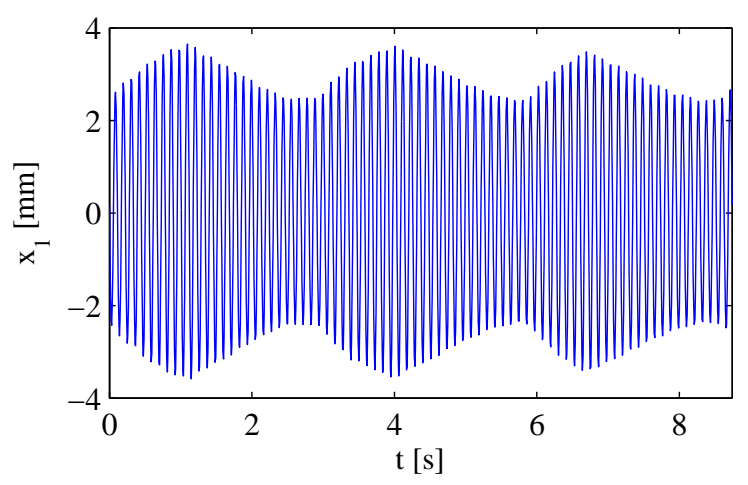

FIGURE 10. Experimental measurement of SMR for $G=0.16 \mathrm{~mm}$ $(A=0.019)$ and $\sigma=1.52$.

trol through SMR occurs on a larger bandwidth. Despite the small mass ratio between the mass of the ball and the mass of the LO, the VI-NES strongly influences the global behavior of the system. Qualitative behavior is explained theoretically, and correspondence between analytical/numerical and experimental results is observed.

\section{CONCLUSION}

In this paper, the dynamic response of a two dof system comprising a Linear Oscillator, subjected to an imposed harmonic displacement, with an embedded Vibro-Impact Nonlinear Energy Sink (VI-NES) is investigated theoretically and experimentally. The VI-NES consist of a ball free to move inside rigid barrier. The collision is modeled using simplified shock theory, and a realistic restitution coefficient is taken into account. Equa-

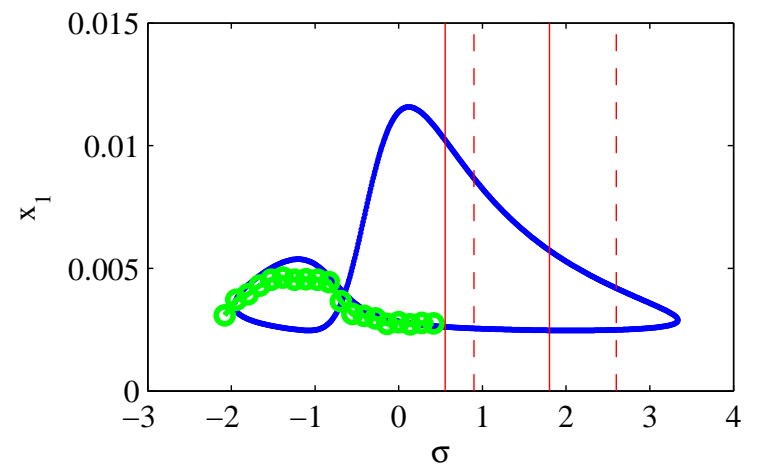

FIGURE 11. Experimental and analytical frequency response curve of the LO for $G=0.14 \mathrm{~mm}(A=0.017)$. Blue lines corresponds to the analytical prediction, green lines represents the experimental measurements. Straight and dashed red lines denotes the experimentally and numerically founded SMR zone.

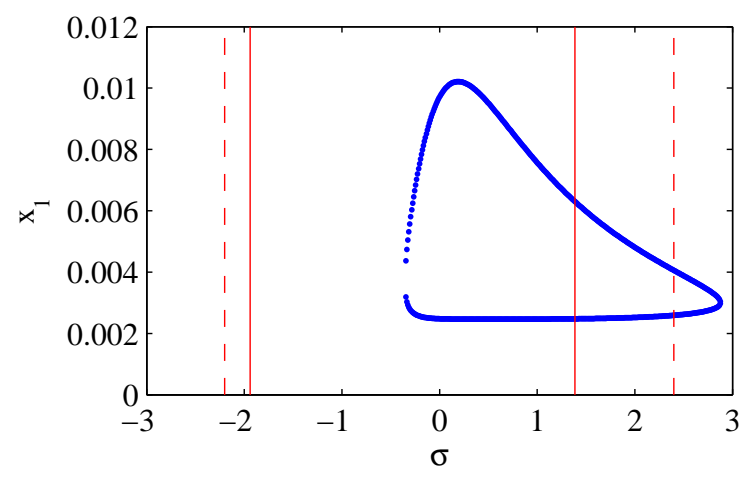

FIGURE 12. Analytical frequency response curve of the LO for $G=0.125 \mathrm{~mm}(A=0.015)$. Blue lines corresponds to the analytical prediction. Straight and dashed red lines denotes the experimentally and numerically founded SMR zone.

tion of motion around the $1: 1$ resonance are analyzed using a multiple scales approach. Symmetric motion with two symmetric impact per cycle are considered. The existence of periodic solution as well as SMR is explained. An experimental device is built. Measurements of frequency response curve for three different forcing amplitudes are presented and compared to analytical predictions. Periodic regimes and also SMR response are observed. The experimentally determined SMR zone is compared to numerical simulation. Even if the model of the NES is a rough approximation of the real motion of the ball, a good agreement between theoretical and experimental results is observed. This type of NES is easy to realize and could be easily integrated in real structure. These results are encouraging to deeper investigate the behavior of this type of NES. 


\section{REFERENCES}

[1] Gendelman, O., Manevitch, L., Vakakis, A., and M'Closkey, R., 2001. "Energy pumping in nonlinear mechanical oscillators: Part i: Dynamics of the underlying hamiltonian systems". Journal of Applied Mechanics, 68(1), pp. 34-41.

[2] Vakakis, A., and Gendelman, O., 2001. "Energy pumping in nonlinear mechanical oscillators: Part ii: Resonance capture". Journal of Applied Mechanics, 68(1), pp. 42-48.

[3] McFarland, D., Bergman, L., and Vakakis, A., 2005. "Experimental study of non-linear energy pumping occurring at a single fast frequency". International Journal of NonLinear Mechanics, 40(6), pp. 891-899.

[4] Gourdon, E., Alexander, N., Taylor, C., Lamarque, C., and Pernot, S., 2007. "Nonlinear energy pumping under transient forcing with strongly nonlinear coupling: Theoretical and experimental results". Journal of Sound and Vibration, 300(3), pp. 522-551.

[5] Kerschen, G., Kowtko, J., McFarland, D., Bergman, L., and Vakakis, A., 2007. "Theoretical and experimental study of multimodal targeted energy transfer in a system of coupled oscillators". Nonlinear Dynamics, 47(1), pp. 285-309.

[6] Gendelman, O., 2004. "Bifurcations of nonlinear normal modes of linear oscillator with strongly nonlinear damped attachment". Nonlinear Dynamics, 37(2), pp. 115-128.

[7] Starosvetsky, Y., and Gendelman, O., 2008. "Strongly modulated response in forced 2 dof oscillatory system with essential mass and potential asymmetry". Physica D: Nonlinear Phenomena, 237(13), pp. 1719-1733.

[8] Gourc, E., Michon, G., Seguy, S., and Berlioz, A., 2012. "Design optimisation of a nonlinear energy sink embedded on a harmonically forced linear oscillator: theoretical and experimental developments". In Proceedings of the ASME, IDETC 2012, VIB-71014, ASME.

[9] Gendelman, O., and Bar, T., 2010. "Bifurcations of selfexcitation regimes in a van der pol oscillator with a nonlinear energy sink". Physica D: Nonlinear Phenomena, 239(3), pp. 220-229.

[10] Lee, Y., Vakakis, A., Bergman, L., McFarland, D., and Kerschen, G., 2007. "Suppressing aeroelastic instability using broadband passive targeted energy transfers, part 1: Theory". AIAA journal, 45(3), pp. 693-711.

[11] Lee, Y., Kerschen, G., McFarland, D., Joel Hill, W., Nichkawde, C., Strganac, T., Bergman, L., and Vakakis, A., 2007. "Suppressing aeroelastic instability using broadband passive targeted energy transfers, part 2: experiments". AIAA journal, 45(10), pp. 2391-2400.

[12] Gendelman, O., Vakakis, A., Bergman, L., and McFarland, D., 2010. "Asymptotic analysis of passive nonlinear suppression of aeroelastic instabilities of a rigid wing in subsonic flow". SIAM Journal on Applied Mathematics, 70(5), pp. 1655-1677.
[13] Vaurigaud, B., Manevitch, L., and Lamarque, C., 2011. "Passive control of aeroelastic instability in a long span bridge model prone to coupled flutter using targeted energy transfer". Journal of Sound and Vibration, 330(11), pp. 2580-2595.

[14] Gourc, E., Seguy, S., Michon, G., and Berlioz, A., 2012. "Delayed dynamical sytem strongly coupled to a nonlinear energy sink: application to machining chatter". In MATEC Web of Conferences, Vol. 1, EDP Sciences.

[15] Gendelman, O., 2008. "Targeted energy transfer in systems with non-polynomial nonlinearity". Journal of Sound and Vibration, 315(3), pp. 732-745.

[16] Lamarque, C., Gendelman, O., Ture Savadkoohi, A., and Etcheverria, E., 2011. "Targeted energy transfer in mechanical systems by means of non-smooth nonlinear energy sink". Acta mechanica, 221(1), pp. 175-200.

[17] Ture Savadkoohi, A., Lamarque, C., and Dimitrijevic, Z., 2012. "Vibratory energy exchange between a linear and a nonsmooth system in the presence of the gravity". Nonlinear Dynamics, 70, pp. 1-11.

[18] Nucera, F., Vakakis, A., McFarland, D., Bergman, L., and Kerschen, G., 2007. "Targeted energy transfers in vibroimpact oscillators for seismic mitigation". Nonlinear Dynamics, 50(3), pp. 651-677.

[19] Nucera, F., Lo Iacono, F., McFarland, D., Bergman, L., and Vakakis, A., 2008. "Application of broadband nonlinear targeted energy transfers for seismic mitigation of a shear frame: Experimental results". Journal of Sound and Vibration, 313(1), pp. 57-76.

[20] Lee, Y., Nucera, F., Vakakis, A., McFarland, D., and Bergman, L., 2009. "Periodic orbits, damped transitions and targeted energy transfers in oscillators with vibroimpact attachments". Physica D: Nonlinear Phenomena, 238(18), pp. 1868-1896.

[21] Gendelman, O., 2012. "Analytic treatment of a system with a vibro-impact nonlinear energy sink". Journal of Sound and Vibration, 331, p. 4599-4608. 